\title{
A Novel Trident Incurvature Shape DGS to Suppress Radiated Emission in High Speed Printed Circuit Board
}

\author{
Anandan Malaiarasan1, Dr. Suresh Kumar Natarajan², Rajeswari Packianathan², \\ Gobinath Arumugam² \\ ${ }^{1}$ Department of Electronics and Communication Engineering, Latha Mathavan Engineering College, \\ Madurai, India \\ ${ }^{2}$ Department of Electronics and Communication Engineering, Velammal College of Engineering and Technology, \\ Madurai, India \\ Email: manandan75@gmail.com, principal@vcet.ac.in, pra@vcet.ac.in, gopigopinath10@gmail.com
}

Received 30 March 2016; accepted 15 April 2016; published 18 August 2016

Copyright (C) 2016 by authors and Scientific Research Publishing Inc.

This work is licensed under the Creative Commons Attribution International License (CC BY). http://creativecommons.org/licenses/by/4.0/

(c) (i) Open Access

\section{Abstract}

As per the entail in wireless communication, the ever increasing switching speeds of digital devices pose significant challenges. Signal quality is more important for high speed products and the signal integrity must ensure reliable transmission where signal integrity is a measure of the quality of an electrical signal. A high speed differential signal will result in signal integrity issues such as crosstalk and radiated emission. One of the solutions to suppress radiated emission is defected ground pattern. This paper introduces a novel trident incurvature shaped defected ground structure to suppress radiated emission that arises in high speed differential signal. The proposed defected ground structure is implemented using Ansoft HFSS simulation tool and its performance is quantified in terms of scattering parameters. The proposed trident incurvature shaped defected ground pattern reduces near end coupling and far end coupling by more than $6 \mathrm{~dB}$ and $2 \mathrm{~dB}$ respectively. It also provides better return loss and insertion loss in the frequency range 1 - $6 \mathrm{GHz}$.

\section{Keywords}

Radiated Emission, Near End Crosstalk, Far End Crosstalk, Return Loss, Insertion Loss, Defected Ground Structure

\section{Introduction}

The rapid growth in wireless communication has been led to the increase in frequency, consistent reduction in

How to cite this paper: Malaiarasan, A., Natarajan, Dr.S.K., Packianathan, R. and Arumugam, G. (2016) A Novel Trident Incurvature Shape DGS to Suppress Radiated Emission in High Speed Printed Circuit Board. Circuits and Systems, 7, 30263037. http://dx.doi.org/10.4236/cs.2016.710258 
size and increase in the circuit complexity. Because of the complexity of the circuits, signal integrity issues posed many challenges to the circuit designers. Signal integrity addresses the issues of timing and the quality of the signal. The timing and quality of the signal are affected by the electrical impact happening at the source end, along the path and the receiving end. The noise problems present in the signal integrity are crosstalk, radiation, ringing, power/ground noise, reflections, attenuation and switching noise. The most prevalent issue in signal integrity is radiation emission which deteriorates the performance of the system. The term radiation emission refers to the unintentional release of electromagnetic energy from a device [1]. The vital reason for radiation emission is current flow and it also occurs when there is a split in a trace. The main cause for radiation emission is common mode noise. Common mode radiation and differential mode radiations occur when a differential signal crosses a slotted reference plane. Common mode current is equal in magnitude and in the same direction, whereas differential mode current is equal in magnitude and in opposite direction. There is an undesired voltage drop in a circuit that causes some parts of the system to be in common mode potential which is very hard to control than differential mode radiation [2]. Several researchers and authors are projected and accordingly varied approaches to suppress common mode radiation on a printed circuit board [3]-[12].

One way to reduce this electromagnetic emission is to use stitching capacitor [3]. It provides return current path of signal lines and degrades the noise or they suppress the signal distortion and decrease radiation emission. But it may cause internal reflection and resonance. By decreasing the space between the ground plane and also the signal traces, the radiation is reduced however it induces the coupling mechanism. So as to decrease the coupling, the ground guard fence was used between the microstrip lines and also the disadvantage of using that would increase the scale of the printed circuit board. It is common to introduce slots within the reference plane to supply the DC isolation [5]. An asymmetrical grounded resonators technique was proposed to suppress the EMI for slot crossing differential signals. These asymmetric resonators with one end shorting to the ground plane provide economical shorting path between the split planes at totally different frequencies. The vias were used at the one end of the resonators to electrically connect the resonators and also the ground plane [7]. In this paper, a novel trident incurvature shaped DGS is introduced to reduce electromagnetic coupling without adding any additional components. Tailed $\mathrm{E}$ and $\mathrm{U}$ shaped structure and by suitably designing it, a good signal quality for the differential component is achieved. The proposed filter is compact because $U$ shaped structure can be inserted between the tailed $\mathrm{E}$ shaped structures.

\section{Radiated Emission on a Printed Circuit Board}

As circuits are reduced in size, a transmission structure was needed that was compatible with circuit construction techniques to provide guided waves over restricted distances. This was realized with a planar transmission line over a ground plane, known as microstrip line. Microstrip line employs a flat strip conductor suspended above a ground plane by a low-loss dielectric material. In the microstrip line, the electromagnetic fields exist partially in the air above the dielectric substrate and partially within the substrate material itself. Figure 1 illustrates the structure of coupled microstrip lines, in which two lines are running parallel. It may be modelled as distributed inductance and capacitance. When the input signal is applied to aggressor line, the electromagnetic coupling generates and crosstalk will be induced in the adjacent victim line [6]. This electromagnetic coupling induced at the close to the adjacent line is called near end coupling and it is induced at the far end of the adjacent line is called far end coupling. The electromagnetic coupling depends on the amount of inductive coupling and capacitive coupling.

The main source of radiated emission in high speed printed circuit board is that the common mode current. The currents on parallel conductors are often divided into two types-common mode current and differential mode current. Differential mode currents are equal in magnitude, however opposite in direction on parallel conductors. The common mode currents are equal in magnitude and in the same direction on parallel conductors. The differential and common mode currents are depicted in Figure 2. In general, an arbitrary current on a parallel conductor system includes of differential and common mode currents. Let the common mode current and differential mode current are represented as $I_{C}$ and $I_{D}$ respectively. Let this on the first conductor be $I_{1}$, which could be the total of common mode and differential mode currents.

$$
I_{1}=I_{C}+I_{D}
$$

Let the current on the second conductor be $I_{2}$, which can be represented as the difference between the common mode and differential mode currents. 


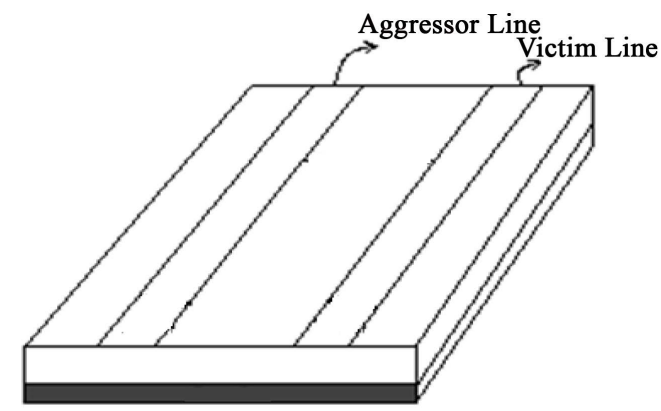

(a)

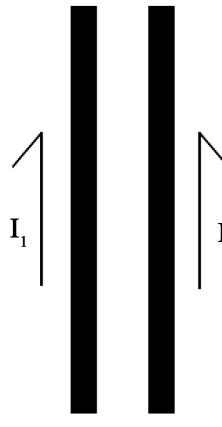

Total Current
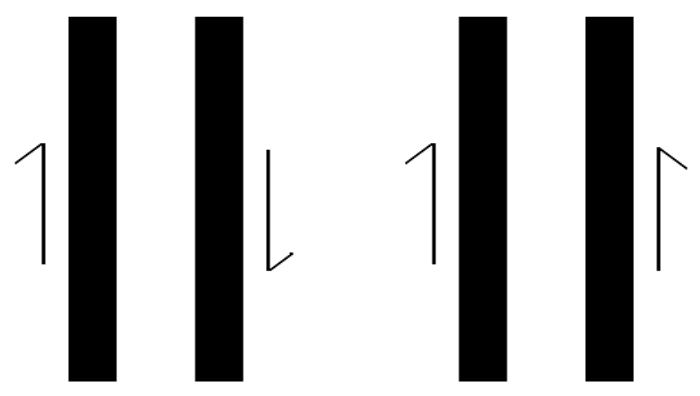

(b)

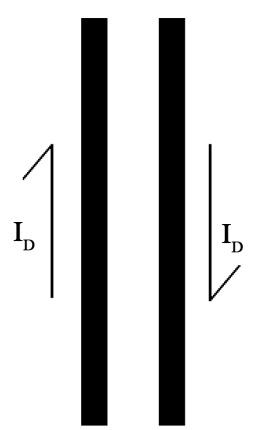

Differential mode Current

(c)

Figure 1. (a) Coupled microstrip line; (b) Differential and common mode currents; (c) Decomposition of total current into common mode and differential mode currents.

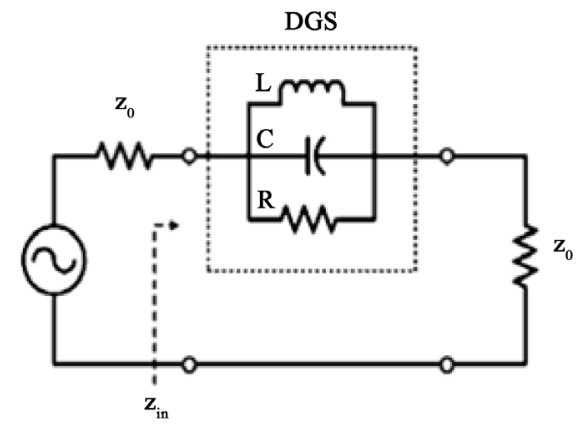

Figure 2. Equivalent circuit model of elemental DGS.

$$
I_{2}=I_{C}-I_{D} \text {. }
$$

Thus, the common mode and differential mode currents are found to be

$$
\begin{gathered}
I_{C}=\left(I_{1}+I_{2}\right) / 2 \\
I_{D}=\left(I_{1}-I_{2}\right) / 2 .
\end{gathered}
$$

\section{Defected Ground Structures}

Defected Ground Structure (DGS) is an intentionally produced defect in the ground plane of a transmission line. The DGS could be a deliberately carven periodic or a non periodic configuration depending on the requirement. The result of defects on a line is that it disturbs the current distribution within the ground plane. Therefore, it changes the effective capacitance and inductance of the line. DGS structures are designed in numerous shapes like square, rectangular, circular, dumbbell shaped, spiral, L-shaped, concentric ring, U-shaped, V-shaped, hair- 
pin DGS, hexagonal DGS and cross shaped DGS are reported in the literature. Every form has its own distinct characteristics that facilitate to improve the performance of a microwave device. DGS is widely used for numerous applications in microwave devices. Because of totally different shapes in etching part, there is a modification in permittivity during return current distribution travelling in the ground trace. The space between ground and signal lines is increased by increasing the capacitance. These include efficiency improvement of power amplifier, frequency control of microstrip antenna and improving working performance of filters and power dividers. DGS has been used to provide frequency selectivity and effective parallel feedback path for an oscillator [3]. Meanwhile, DGS reduces the mutual coupling between the antenna array elements. DGS resonators are applied in a very few variety of microstrip circuits to scale back the quality and improve the performance [8].

The circuit parameters of the equivalent circuit are extracted from the simulated scattering parameters as

$$
\begin{gathered}
C=\frac{w_{c}}{2 Z_{0}\left(w_{0}^{2}-w_{c}^{2}\right)} \\
L=\frac{1}{4 \pi^{2} f_{0}^{2} C} \\
R=\frac{2 Z_{0}}{\sqrt{\frac{1}{\left|S_{11}\left(w_{0}\right)\right|^{2}}-\left(2 Z_{0}\left(w_{0} C-\frac{1}{w_{0} L}\right)\right)^{2}-1}} .
\end{gathered}
$$

Here, $w_{0}$ is the angular resonance frequency, $w_{c}$ is the 3-dB cutoff angular frequency, and $Z_{0}$ is that the characteristic impedance of the microstrip line. The characteristics of the U-slot DGS like the resonance frequency, rejection bandwidth, and factor are reliant on the structural parameters of the deformity. The scale of slot length, slot width, and distance between two slots are the parameters ever-changing those exchange attributes of the DGS. Because the slot length will increase, both the equivalent capacitance and equivalent inductance increase. As the slot length increases, each the equivalent capacitance and equivalent inductance will increase. As the slot width grows, the equivalent capacitance decreases and therefore the equivalent inductance will increase, the resonance frequency slowly decreases because of the inductance increase [10], it is affirmed that the processed part increases as the slot width decreases.

The H shaped DGS is made to locate at the ground plane as shown in Figure 3. The diminishment of radiation discharge can be achieved by utilizing $\mathrm{H}$ molded ground plane pattern. The carving region in this kind of ground plane is more in this structure, so it reduces radiated emission by reducing capacitive coupling [5]. This Structure is simulated and these results for the s-parameters were presented.

The designed U-slot DGS gives the attributes, for example, the resonance frequency, rejection bandwidth, and the elements which are reliant on the structural parameters of the imperfection. This shows maximum effective capacitance when the slot width decreases, however, it involves more space.

Two U shaped and one $\mathrm{H}$ shaped structure on ground plane forms three mutually coupled resonators. Since, these three resonators are located closely, mutual coupling between any two resonators are taken. Mutual

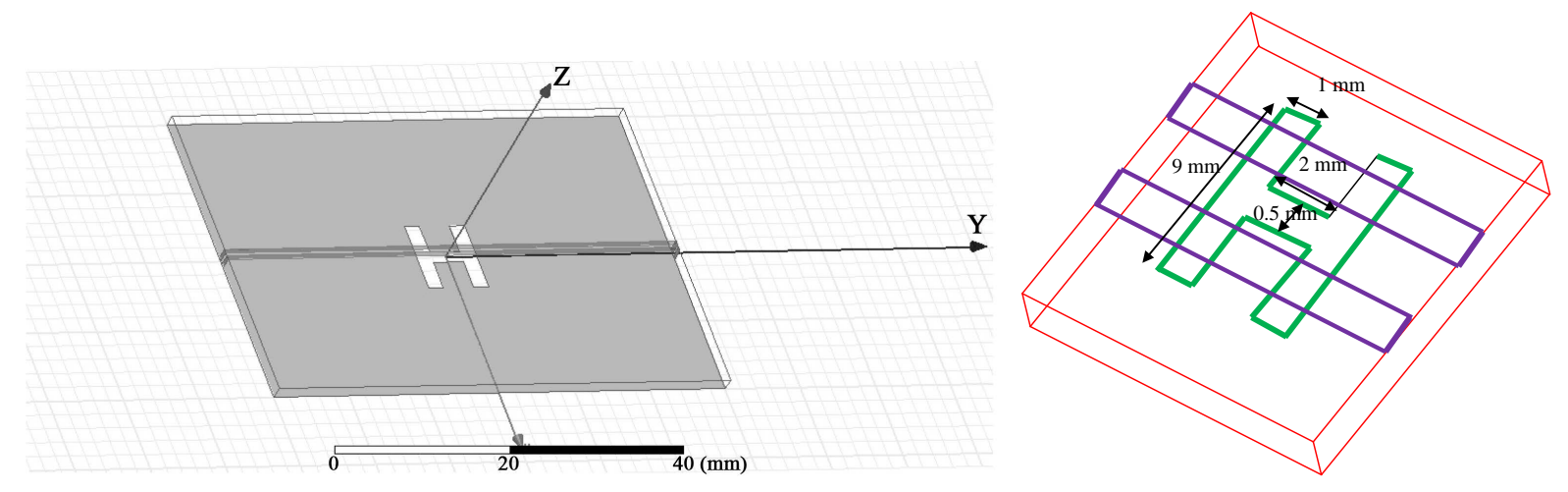

Figure 3. Simulated structure of H shape. 
coupling is synchronous for two identical $\mathrm{U}$ shaped resonators and is asynchronous between $\mathrm{U}$ and $\mathrm{H}$ shaped resonators [10]. It is observed that the common mode noise can be reduced more than the alternate structures

Trident incurvature shaped DGS is used for proposed structure. Trident shape utilized as a part of the proposed structure has a measurement of $9 \mathrm{~mm}$ length and $4.5 \mathrm{~mm}$ in the expansiveness of corner lines, with thickness of $0.3 \mathrm{~mm}$. The incurvature shape is coupled with the trident shape which has $l \times b$ as $6 \times 3$. By varying the dimensions of the structure we obtained optimum results. Return loss, insertion loss, near end coupling, far end coupling was measured. Through this proposed structure, high return loss is obtained, which is much better than the conventional structures. From this structure we have acquired the perfect result. The major advantage of using this structure is it has a minimum loop area than different DGS structure and also it has augmented in the effective capacitance.

\section{Results and Discussions}

The various DGS structure shown in Figures 3-6 were etched on ground plane and a dielectric thickness of 1.6 $\mathrm{mm}$ with with $\varepsilon_{\mathrm{r}}=4.4$. The physical dimensions mentioned in Figures 3-6 were considered for the simulations.

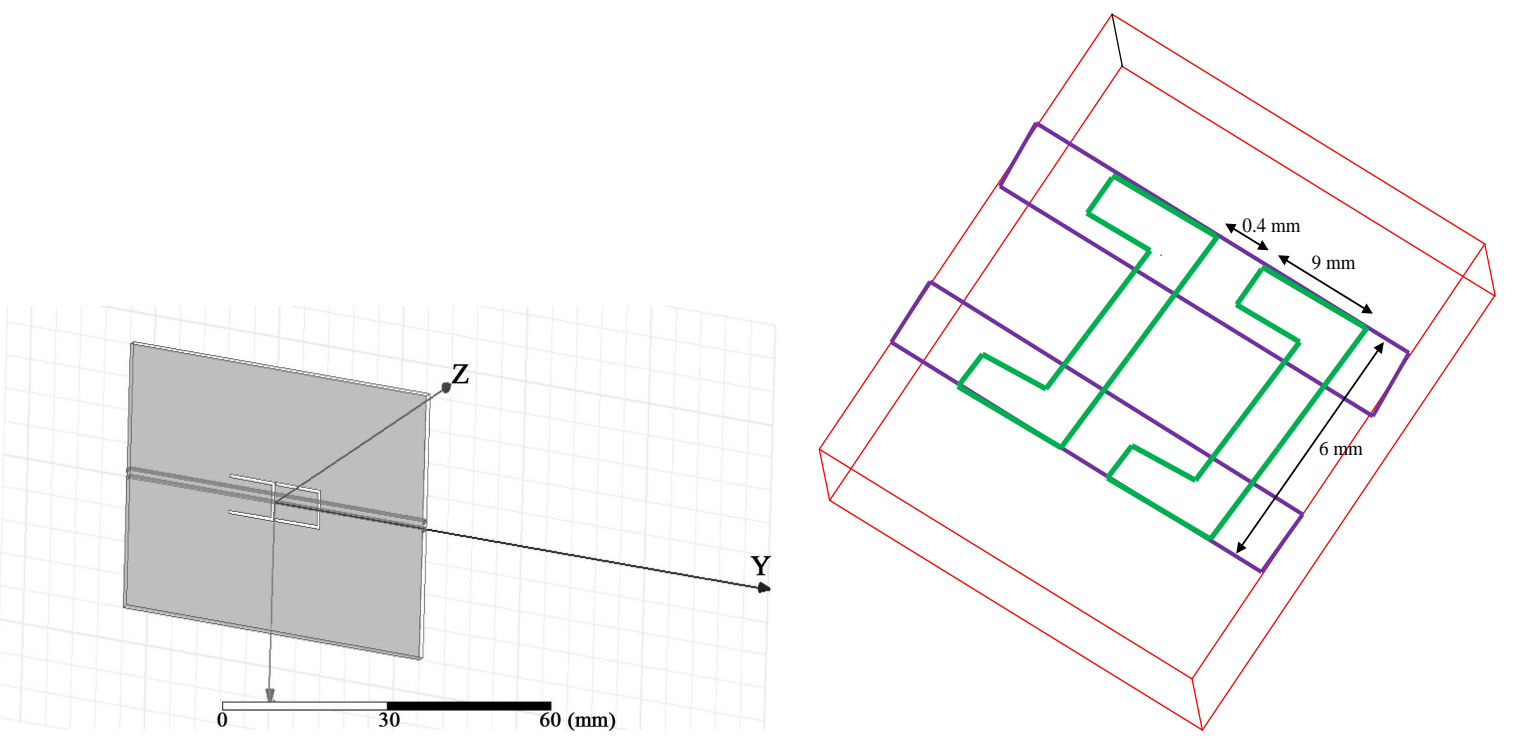

Figure 4. Simulated structure of cascaded U-shape.
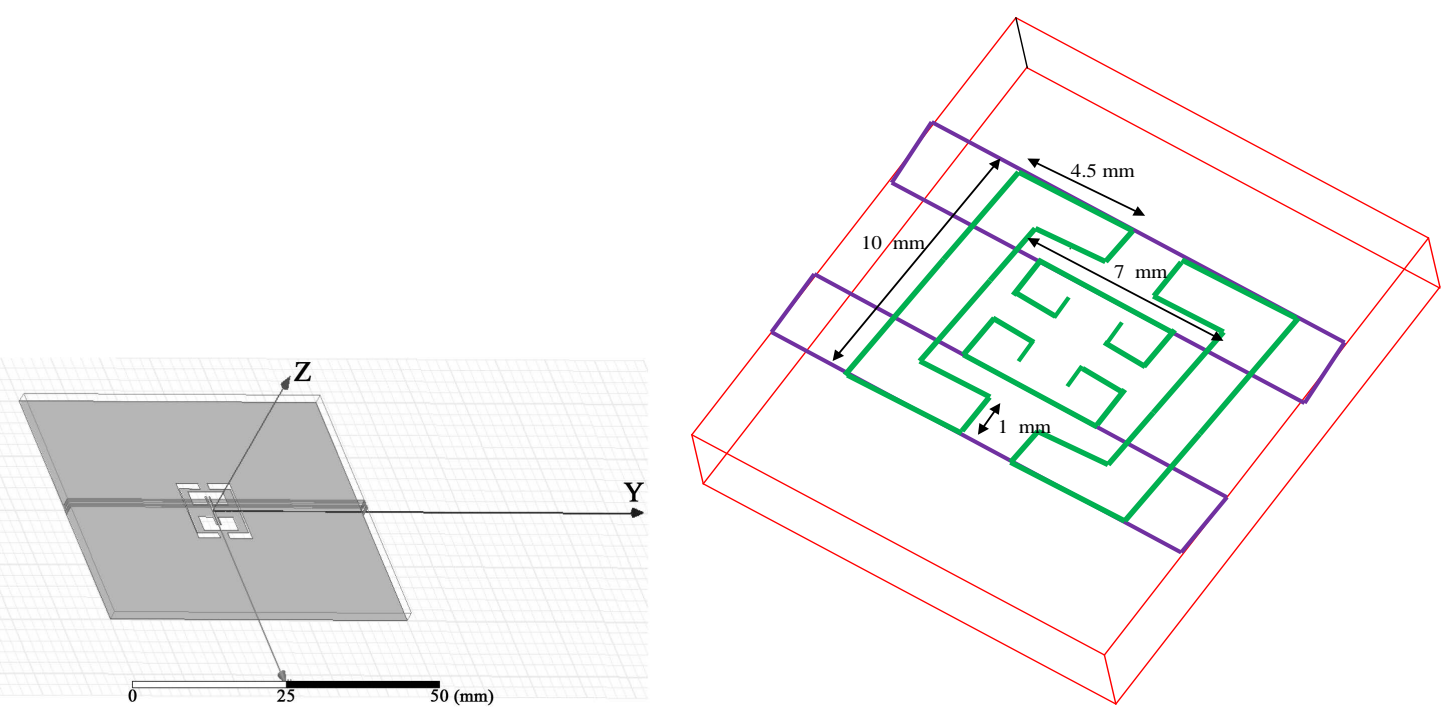

Figure 5. Simulated structure of UH shaped DGS. 


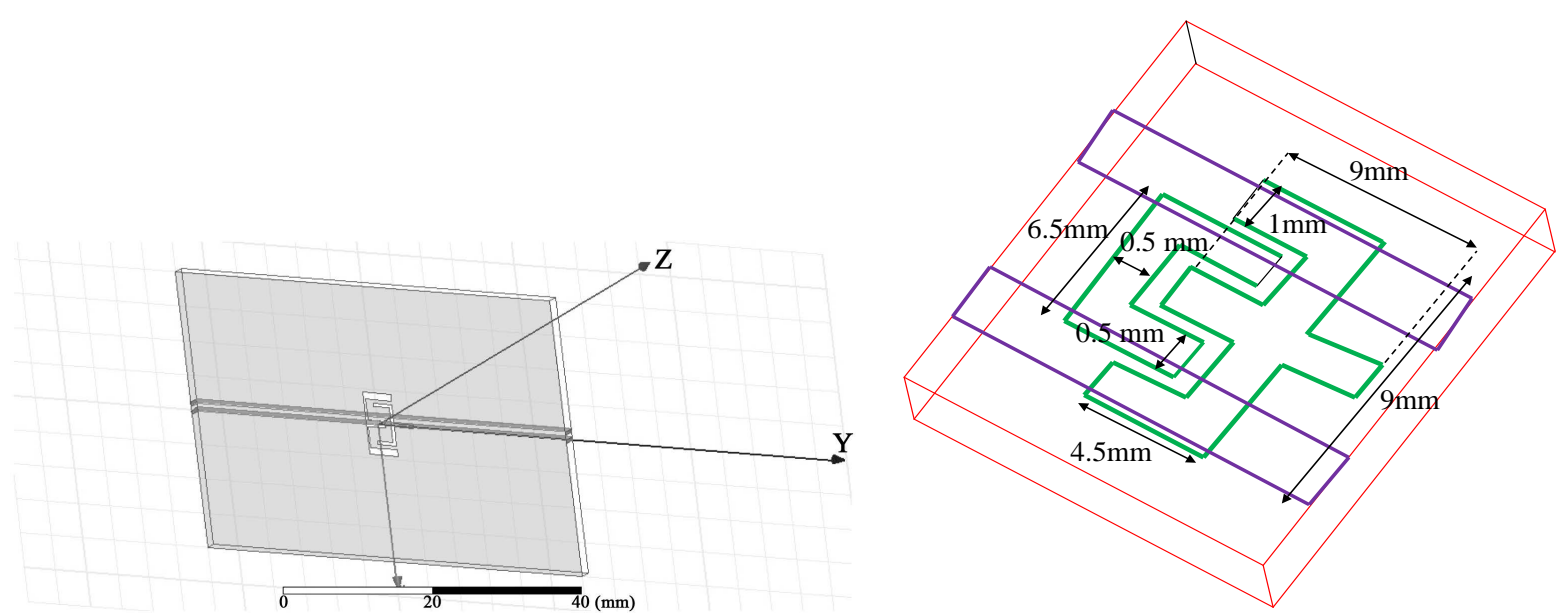

Figure 6. Simulated structure of proposed DGS.

The simulation results were obtained with the help of Ansoft HFSS over the frequency range from $0-6 \mathrm{GHz}$ [13]. The H shaped DGS had total length and breadth of $9 \mathrm{~mm}$ and $4 \mathrm{~mm}$. The near end coupling and far end coupling, return loss, insertion loss and E \& H-field distribution are given in Figure 7.

The PCB used here for cascaded U shaped DGS had a dimension of $60 \mathrm{~mm} \times 40 \mathrm{~mm}$. The length and width of the differential line are around $60 \mathrm{~mm} \times 0.545 \mathrm{~mm}$. The E-field pattern and H-field pattern for cascaded shaped ground plane structure are shown in Figure 8.

The dimension of the UH ground plane structure was about $10 \times 10 \mathrm{~mm}$. The $\mathrm{H}$ shaped ground structure was surrounded by two $\mathrm{U}$ shaped ground structures. This provides a minimum return current path. Figure 9 showed the performance of UH shaped DGS. Compared with cascaded U shape DGS, it reduces far end coupling at the cost of increase in near end coupling.

Trident incurvature shaped ground plane structure, reduce radiation rapidly for the range of frequency up to 6 GHz. The outline illustration in a microstrip DGS structure gives a decent understanding between the deliberate and reenacted recurrence reaction of the channel and checks in this manner the proposed system. Subsequently, it is normal that the proposed structures with its smallness, effortlessness and extensive stop band qualities will be an in number contender for applications in different incorporated microwave circuits and in addition different sorts of channels. The graph showed the S-parameters of the proposed structure which has anear end coupling of -22.0416 , far end coupling of -8.4398 , Insertion loss of -7.09 , Return loss of -13.872 . Compared to conventional structures the results obtained for the proposed structure has a reduced near end and far end coupling (Figure 10).

Table 1 summarizes the performance of conventional DGS and proposed DGS in frequency domain. It shows proposed DGS reduces both near end coupling and far end coupling. And also the reflection loss is very less compared with other structures. The time domain analysis is also done and it is given in the Table 2. The proposed DGS reduces near end coupling voltage more than $40 \mathrm{mV}$ than other existing DGS.

\section{Conclusion}

The coupling between traces in conventional microstrip line examined by utilizing refreshed method is called DGS. It is a least complex strategy which lessens the measure of the printed circuit board. The imperfect structures give more prominent execution which is not created by other conventional technologies. The concern is to study the improvement in the radiation emission of the microstrip structure. Outline and re-enactment will be focused on finding more suitable structures for the decrease of radiation emission to the bigger degree and the structure is upgraded by a proficient improved procedure. Simulation and furthermore, estimation results indicate great concurrence with one another, checking the precision of the proposed circuit model furthermore, the prevalent execution of the proposed designed ground structure. Thus, at $6 \mathrm{GHz}$, the return loss shows a reduction of about $8 \mathrm{~dB}$ and near end coupling about $15 \mathrm{~dB}$. The deliberate results are in great concurrence with the simulated results. 


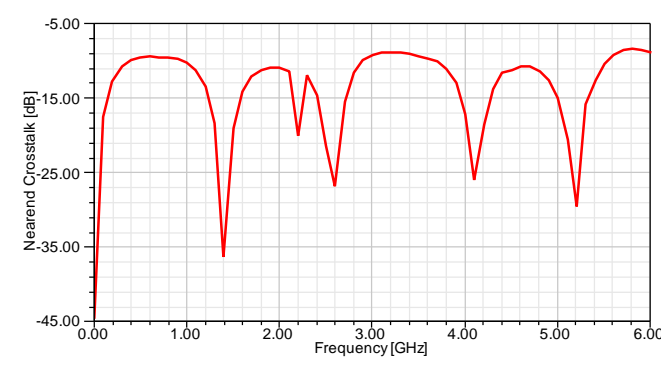

(a)

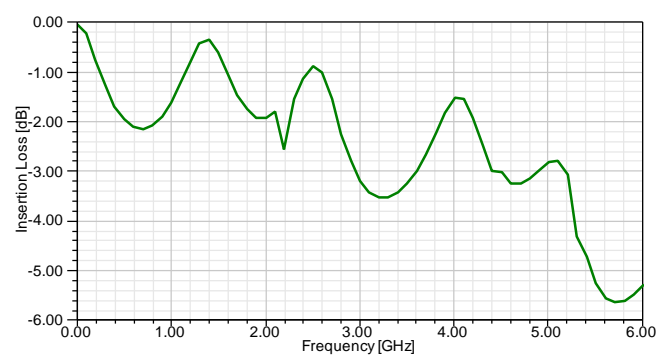

(c)

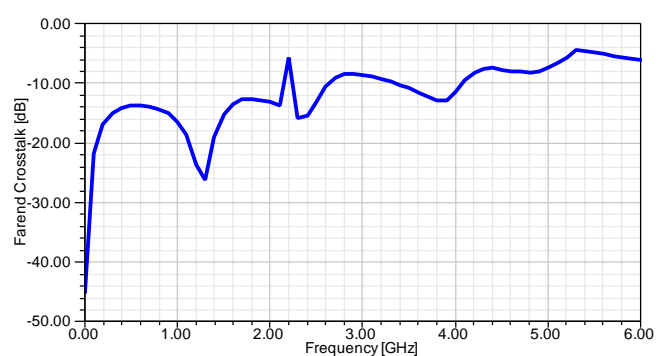

(b)

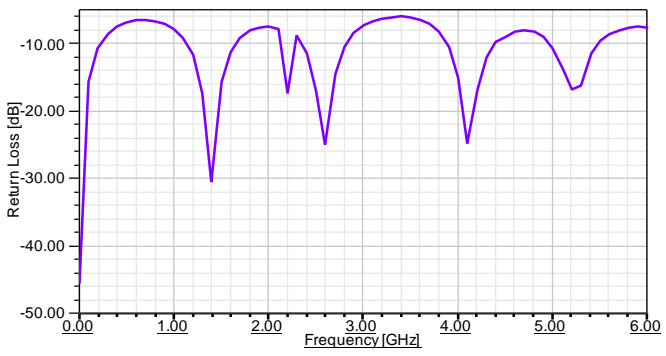

(d)

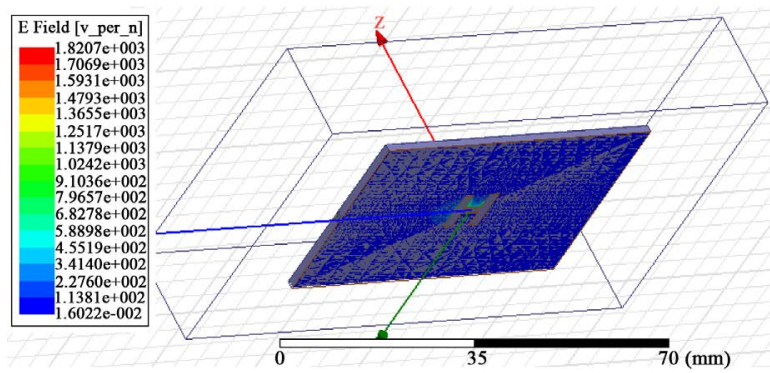

(e)

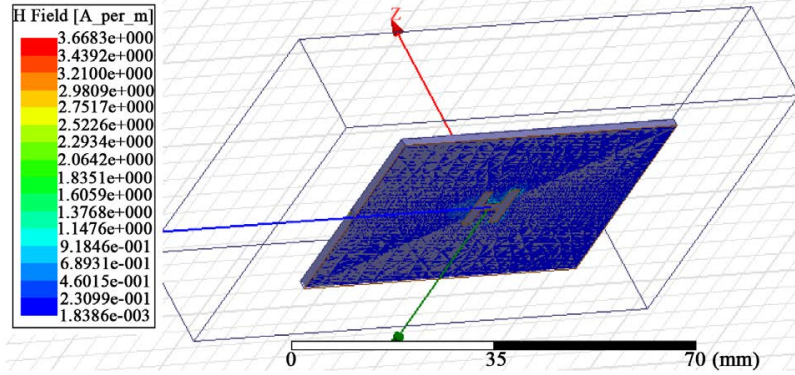

(f)

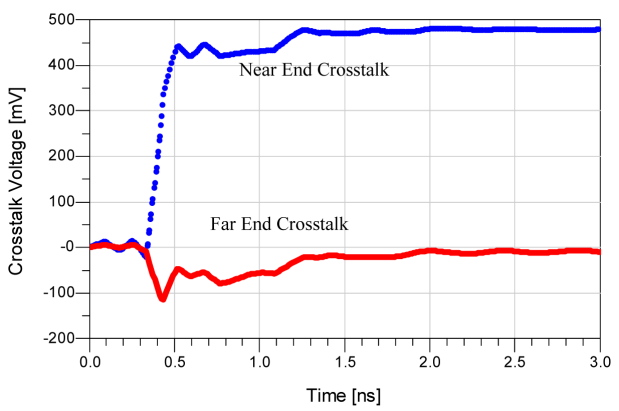

(g)

Figure 7. Simulated results of H shaped DGS: (a) Near end coupling; (b) Far end coupling; (c) Insertion loss; (d) Return loss; (e) E-field distribution; (f) H-field distribution. 


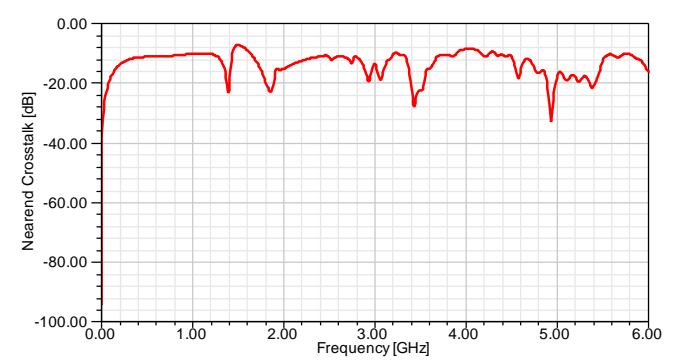

(a)

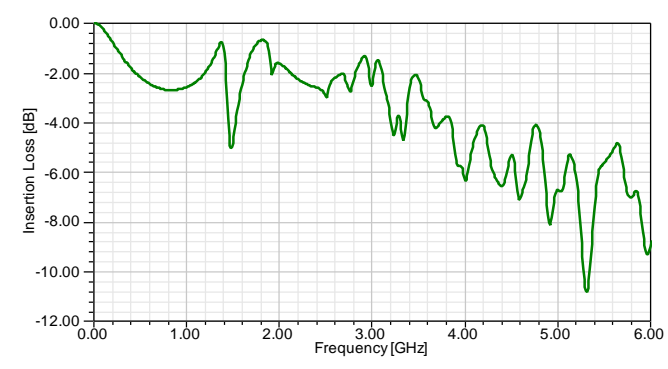

(c)

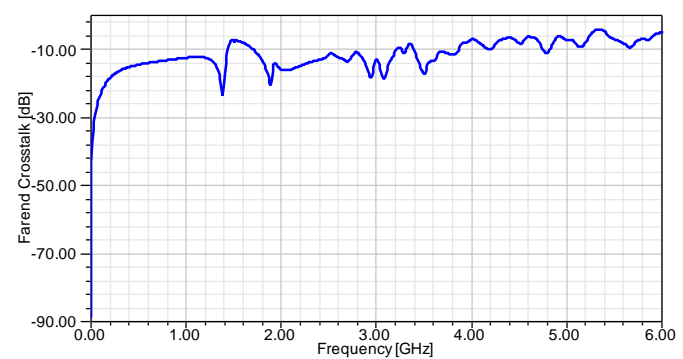

(b)

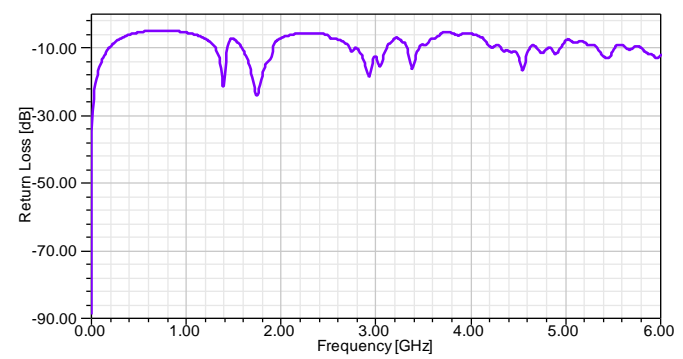

(d)

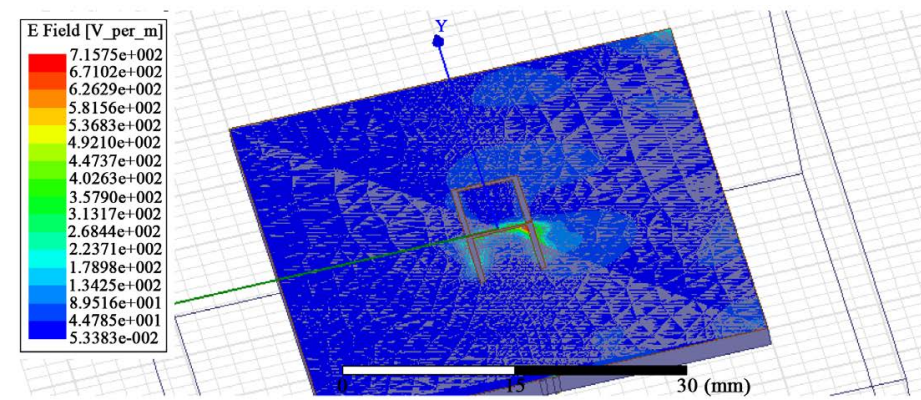

(e)

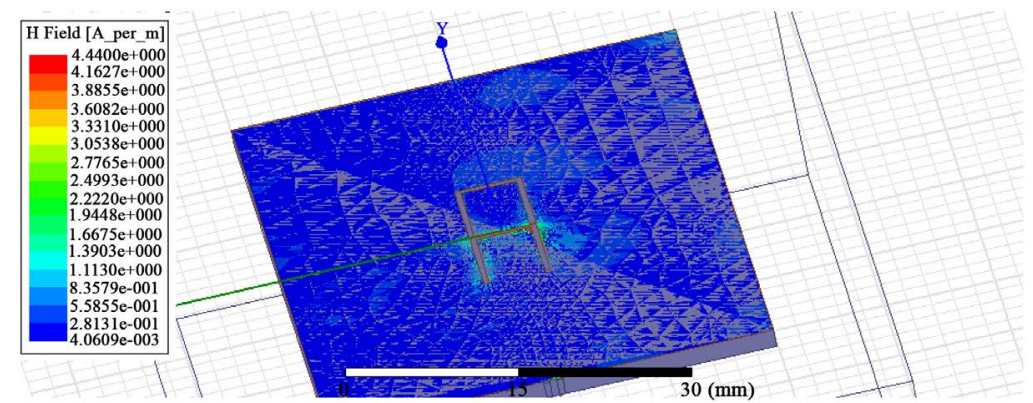

(f)

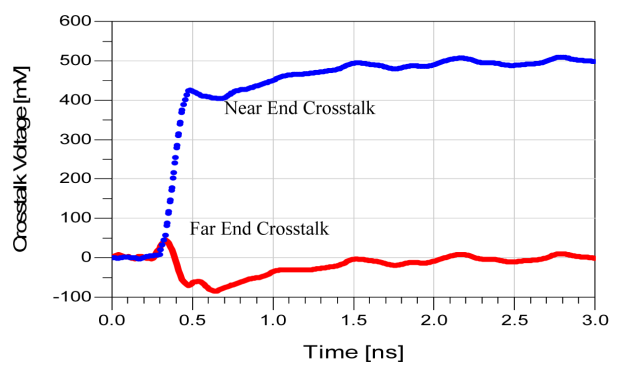

(g)

Figure 8. Simulated results of U shaped DGS: (a) Near end coupling; (b) Far end coupling; (c) Insertion loss; (d) Return loss; (e) E-field distribution; (f) H-field distribution. 


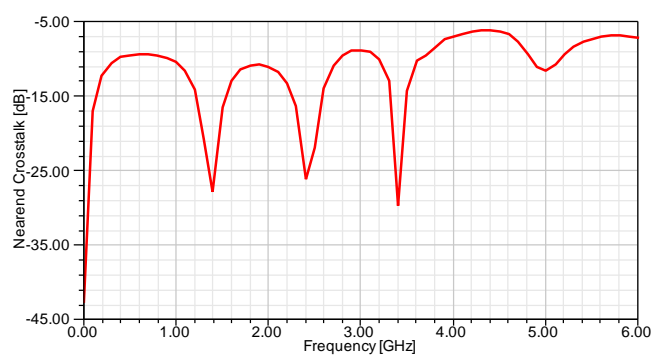

(a)

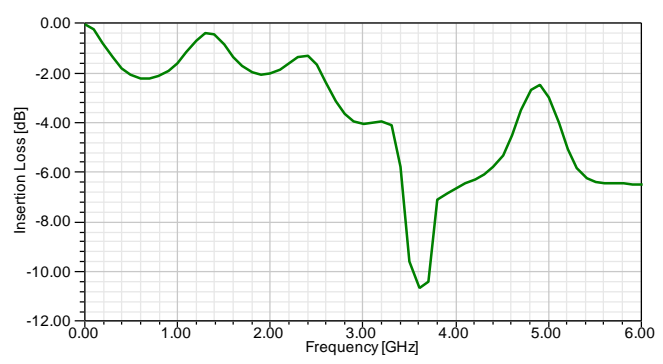

(c)

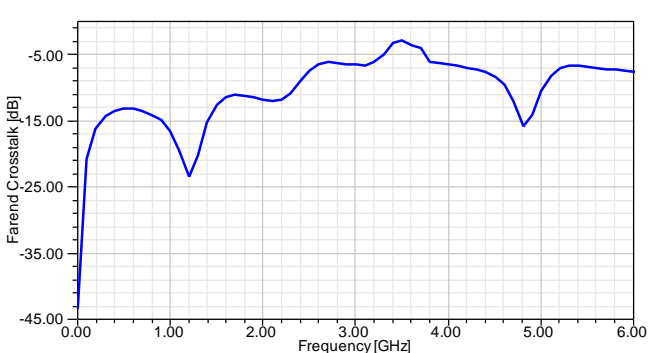

(b)

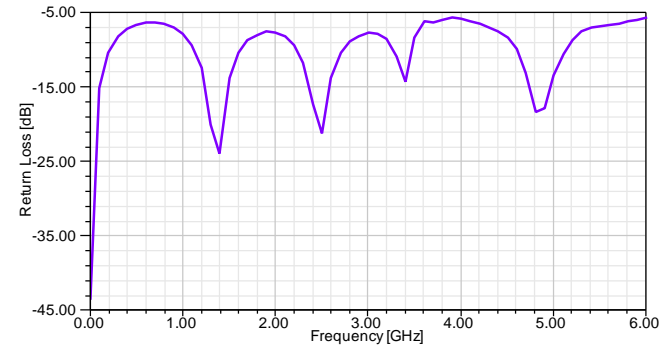

(d)

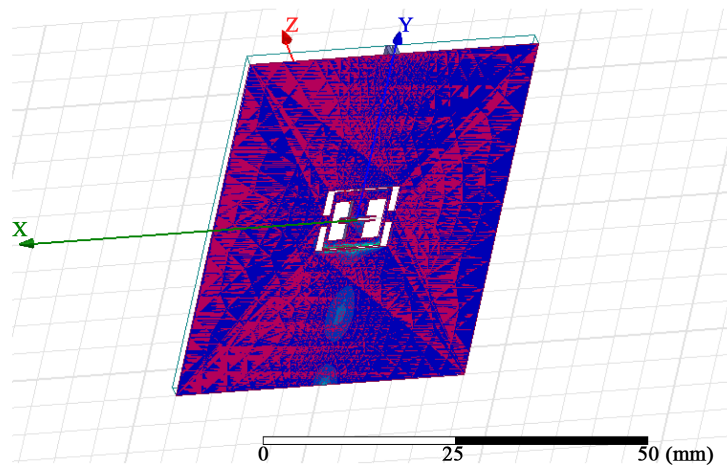

(e)

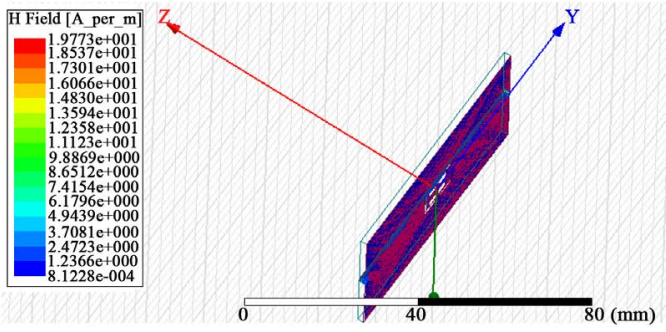

(f)

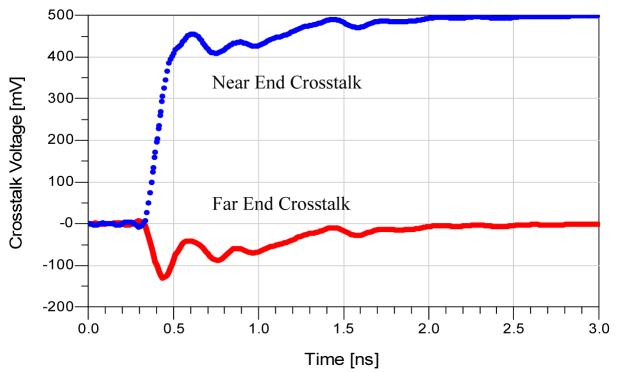

(g)

Figure 9. Simulated results of UH shaped DGS: (a) Near end coupling; (b) Far end coupling; (c) Insertion loss; (d) Return loss; (e) E-field distribution; (f) H-field distribution. 


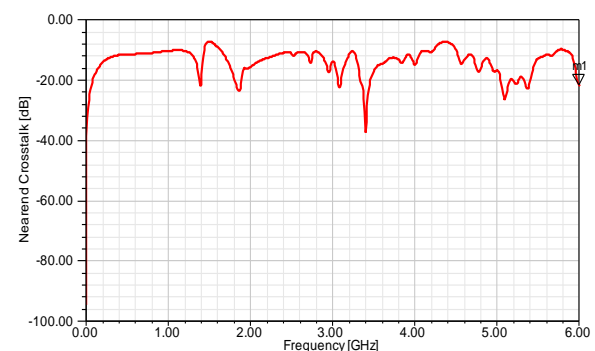

(a)

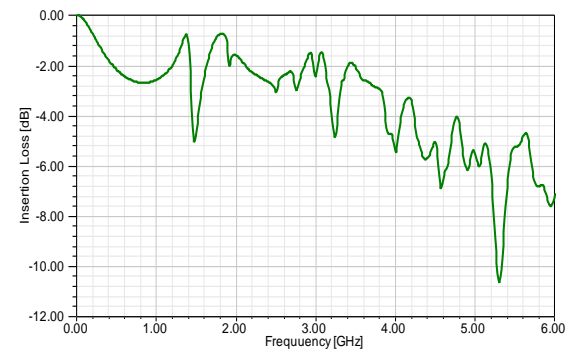

(c)

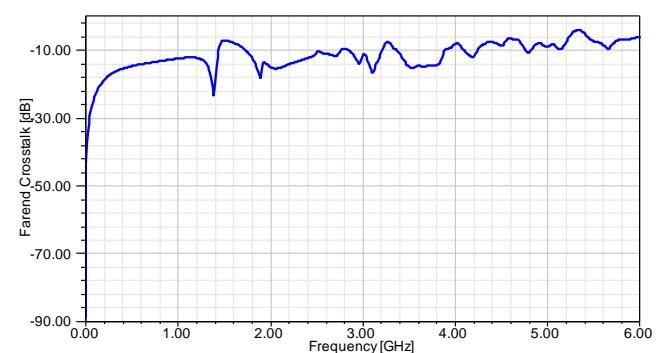

(b)

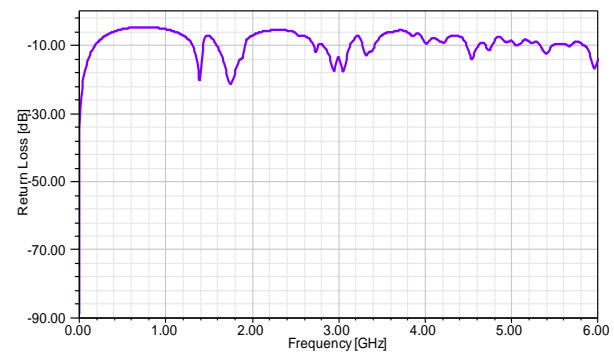

(d)

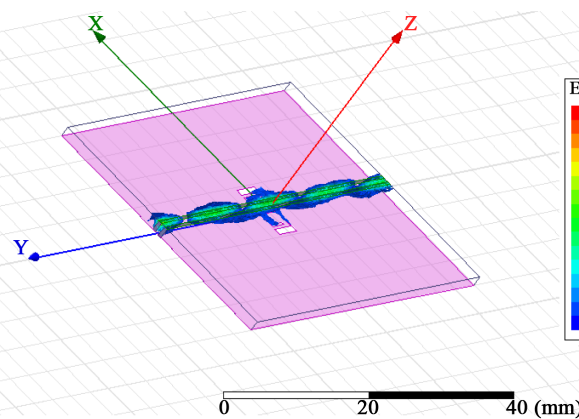

(e)

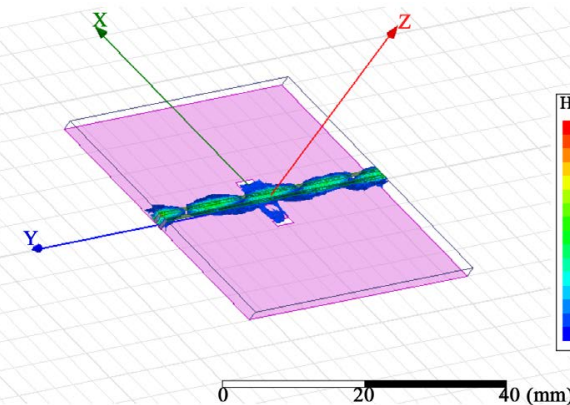

(f)

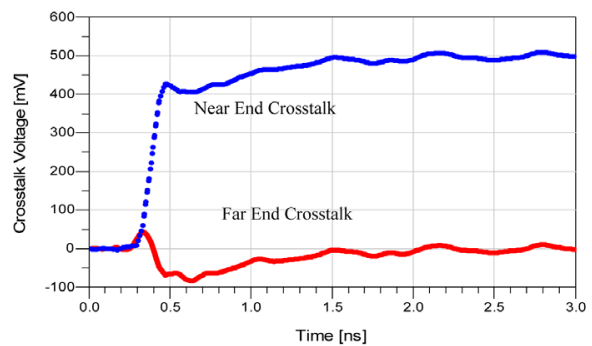

(g)

Figure 10. Simulated results of proposed DGS: (a) Near end coupling; (b) Far end coupling; (c) Insertion loss; (d) Return loss; (e) E-field distribution; (f) H-field distribution. 
Table 1. Performance comparison of conventional and proposed structures in frequency domain.

\begin{tabular}{ccccc}
\hline Structures & Near End Coupling $\left(\mathrm{S}_{13}\right)$ & Far End Coupling $\left(\mathrm{S}_{14}\right)$ & Insertion Loss $\left(\mathrm{S}_{12}\right)$ & Return Loss $\left(\mathrm{S}_{11}\right)$ \\
\hline H Shaped DGS & -8.93 & -6.26 & -5.289 & -7.6845 \\
Cascaded U Shaped DGS & -16.08 & -5.31 & -9.159 & -11.77 \\
UH Shaped DGS & -7.3411 & -7.726 & -6.5150 & -5.7829 \\
Proposed DGS & -22.0416 & -8.4398 & -7.09 & -13.872 \\
\hline
\end{tabular}

Table 2. Performance comparison of conventional and proposed structures in time domain.

\begin{tabular}{ccc}
\hline Structures & \multicolumn{3}{c}{ Time Domain } & \\
\cline { 2 - 3 } & $\mathrm{V}_{\text {next }}(\mathrm{mV})$ & $\mathrm{V}_{\text {fext }}(\mathrm{mV})$ \\
\hline H Shaped DGS & 450.956 & -120.06 \\
Cascaded U Shaped DGS & 420.20 & -95.65 \\
UH Shaped DGS & 440.65 & -110.50 \\
Proposed DGS & 400.12 & -85.02 \\
\hline
\end{tabular}

\section{Acknowledgements}

The authors would like to thank Velammal College of Engineering and Technology, Madurai for providing the support.

\section{References}

[1] Paul, C. (2006) Introduction to Electromagnetic Compatibility. Wiley-Interscience, New York.

[2] Ott, H.W. (1988) Noise Reduction Techniques in Electronic Systems. Wiley-Interscience, New York.

[3] Kim, J., Lee, H. and Kim, J. (2005) Effects on Signal Integrity and Radiated Emission by Split Reference Plane on High-Speed Multilayer Printed Circuit Boards. IEEE Transactions on Advanced Packaging, 28, No. 4.

[4] Zeng, Z.B., Zhuang, Y.Q., Shen, Z.N., Yao, Y.D. and Xiang, X. (2014) A Novel Simple Wide Band Common-mode Suppression Filter. IEICE Electronics Express, 11, No. 17.

[5] Chuang, H.-H. and Wu, T.-L. (2010) A Novel Ground Resonator Technique to Reduce Common-Mode Radiation on Slot-Crossing Differential Signals. IEEE Microwave and Wireless Components Letters, 20, 660-662.

[6] Naser-Moghadasi, M., Alamolhoda, M. and Rahmati, B. (2011) Spurious-Response Suppression in Microstrip Parallel-Coupled Bandpass Filters by Using Defected Microstrip Structures. 1st Annual International Interdisciplinary Conference (AIIC 2013), Azores, Portugal, 24-26 April 2013.

[7] Chuang, H.-H. and Wu, T.-L. (2011) Suppression of Common-Mode Radiation and Mode Conversion for SlotCrossing GHz Differential Signals Using Novel Grounded Resonators. IEEE Transactions on Electromagnetic Compatibility, 53, 429-436.

[8] Mandal, M.K. and Sanyal, S. (2006) A Novel Defected Ground Structure for Planar Circuits. IEEE Microwave and Wireless Components Letters, 16, No. 2.

[9] Liu, W.-T., Tsai, C.-H., Han, T.-W. and Wu, T.-L. (2008) An Embedded Common-Mode Suppression Filter For GHz Differential Signals Using Periodic Defected. IEEE Microwave and Wireless Components Letters, 18, No. 4.

[10] Chuang, H.-H. and Wu, T.-L. (2011) Suppression of Common Mode Radiation and Mode Conversion for Slot Crossing GHz Differential Signals Using Novel Grounded Resonators. IEEE Transactions on Electromagnetic Compatibility, 53, No. 2.

[11] Wu, T.-L., Tsai, C.-H., Wu, T.-L. and Itoh, T. (2009) A Novel Wideband Common-Mode Suppression Filter for Gigahertz Differential Signals Using Coupled Patterned Ground Structure. IEEE Transactions on Microwave Theory and Techniques, 57, 848-855. 
[12] Kim, C.-S., Park, J.-S., Ahn, D. and Lim, J.-B. (2000) A Novel 1-D Periodic Defected Ground Structure for Planar Circuits. IEEE Microwave and Guided Wave Letters, 10, No. 4.

[13] Ansoft HFSS User’s Guide-High Frequency Structure Simulator. Ansoft, Pittsburgh, PA.

\section{Submit or recommend next manuscript to SCIRP and we will provide best service for you:}

Accepting pre-submission inquiries through Email, Facebook, LinkedIn, Twitter, etc.

A wide selection of journals (inclusive of 9 subjects, more than 200 journals)

Providing 24-hour high-quality service

User-friendly online submission system

Fair and swift peer-review system

Efficient typesetting and proofreading procedure

Display of the result of downloads and visits, as well as the number of cited articles

Maximum dissemination of your research work

Submit your manuscript at: http://papersubmission.scirp.org/ 